\title{
On the Topological Entropy of Subshifts of Finite Type on Free Semigroups
}

\author{
Jung-Chao Ban and Chih-Hung Chang*
}

\begin{abstract}
In this paper, we provide an effective method to compute the topological entropies of $G$-subshifts of finite type ( $G$-SFTs) with $G=F_{d}$ and $S_{d}$, the free group and free semigroup with $d$ generators respectively. We develop the entropy formula by analyzing the corresponding systems of nonlinear recursive equations (SNREs). Four types of SNREs of $S_{2}$-SFTs, namely the types $\mathbf{E}, \mathbf{D}, \mathbf{C}$ and $\mathbf{O}$, are introduced, and we could compute their entropies explicitly. This enables us to give the complete characterization of $S_{2}$-SFTs on two symbols. That is, the set of entropies of $S_{2}$-SFTs on two symbols is equal to $\mathbf{E} \cup \mathbf{D} \cup \mathbf{C} \cup \mathbf{O}$. The methods developed in $S_{d^{-}}$SFTs will also apply to the study of the entropy theory of $F_{d}$-SFTs. The entropy formulae of $S_{d^{-}}, F_{d^{-}}$golden mean shifts and $k$-colored chessboards are also presented herein.
\end{abstract}

\section{Introduction}

A classical dynamical system is a pair $(X, T)$ in which $X$ is a phase space and $T: \mathbb{R}^{+} \times X \rightarrow$ $X$ is a function that describes the evolution of elements of $X$. If a dynamical system is hyperbolic, the symbolic dynamical system is an essential and useful tool to investigate the original system [5, 17]. There is a partition $\mathcal{P}=\left\{P_{1}, \ldots, P_{m}\right\}$ of $X$ and a coding map $\pi: X \rightarrow\{1, \ldots, m\}^{\mathbb{N} \cup\{0\}}$ such that $\sigma \circ \pi=\pi \circ T$, where $\sigma:\left(\xi_{n}\right)_{n=0}^{\infty} \mapsto\left(\xi_{n+1}\right)_{n=0}^{\infty}$ is the shift map on $\pi(X)$. One could gain almost all information of $(X, T)$ from $(\pi(X), \sigma)$. The system $(\pi(X), \sigma)$ is also called a $\mathbb{Z}$-symbolic dynamical system. A natural extension of the $\mathbb{Z}$-symbolic dynamical system is the $G$-dynamical system, a pair $(X, T)$ such that $X$ is a topological space, and $T: G \times X \rightarrow X$ is a group action on $X$. These spaces appear naturally as discrete versions of dynamical systems (see [10] and the references therein). A pair $(X, \sigma)$ is called a $G$-subshift if there exists a finite set $\mathcal{A}$ such that $X$ is a $\sigma$-invariant closed subset of $\mathcal{A}^{G}$, where $\sigma: G \times X \rightarrow X$ is defined by $\left(\sigma^{g} x\right)(h)=x\left(g^{-1} h\right)$. A $G$-dynamical system is topologically conjugate to a $G$-subshift if and only if it is zerodimensional and expansive $(G=\mathbb{Z}[8]$, general group [10]), it is therefore of interest to

Received March 6, 2020; Accepted December 21, 2020.

Communicated by Cheng-Hsiung Hsu.

2020 Mathematics Subject Classification. 37A35, 37B10, 92B20.

Key words and phrases. G-SFT, entropy, SNRE, Type E, D, O, C.

This work is partially supported by the Ministry of Science and Technology, Taiwan (Contract No. MOST 109-2115-M-004-002-MY2 and 109-2115-M-390-003-MY3).

*Corresponding author. 
study the $G$-subshifts. This paper aims to investigate the $F_{d^{-}}$and $S_{d^{-}}$-subshifts, where $F_{d}$ and $S_{d}$ are the free group and free semigroup, respectively.

Let $F_{d}$ be a free group that is generated by $S=\left\{s_{1}, \ldots, s_{d}\right\}$, and $\mathcal{A}$ is a finite symbol set. A coloring is a function $f: F_{d} \rightarrow \mathcal{A}$, and a set $\mathcal{F} \subset \mathcal{A} \times S \times \mathcal{A}$ is called forbidden transitions. A coloring $f$ contains $\left(a, s_{i}, b\right) \in \mathcal{F}$ if there exists $g \in F_{d}$ such that $f(g)=a$ and $f\left(g s_{i}\right)=b$. Denote by $X_{\mathcal{F}}$ the set of colorings that do not contain any transitions in $\mathcal{F}$. Since $\mathcal{F}$ is finite, we call $\left(X_{\mathcal{F}}, \sigma\right)$ a $G$-SFT, where the action $\sigma$ is given by $\left(\sigma_{i} f\right)(g)=$ $f\left(s_{i}^{-1} g\right)$ for $g \in F_{d}$ and $f \in X_{\mathcal{F}}, 1 \leq i \leq d$. A pattern with support $\Omega \subset F_{d}$ is an element $p \in \mathcal{A}^{\Omega}$, and we write $\operatorname{supp}(p)=\Omega$. Let $n \in \mathbb{N}$, we denote by $E_{n}$ the set of elements in $G$ whose length is less than or equal to $n$ and denote by $B_{n}\left(X_{\mathcal{F}}\right)$ the set of all possible colorings of $X_{\mathcal{F}}$ in $E_{n}$. The topological entropy (entropy for short) of $X_{\mathcal{F}}$ is defined as

$$
h\left(X_{\mathcal{F}}\right)=\limsup _{n \rightarrow \infty} \frac{\ln \left|B_{n}\left(X_{\mathcal{F}}\right)\right|}{\left|E_{n}\right|}
$$

where the limit 1.1 exists for $G=S_{d}$ due to the recent result of Petersen-Salama (see 15. Theorem 5.1]). The definition of topological entropy is motivated from the classical definition of topological entropy of $G$-shift spaces whenever $G$ is an amenable group. For the case $G=\mathbb{Z}^{d}$, the topological entropy is defined as the growth rate of number of patterns on the $n$-cube (or $n$-ball), which is one of the most considered Følner sequences. Nonetheless, non-amenability of $G=F_{d}, S_{d}$ implies that Følner sequences do not exist. The limit computed here may depend on the exhausting sequence in contrast to the amenable case. It is an interesting problem to find the exact value or formula of (1.1). For the case where $G=\mathbb{Z}^{d}$ with $d=1$, the entropy formulae of $\mathbb{Z}$-SFTs and its algebraic characterization are given by D. Lind [11,12]. Precisely, the nonzero entropies of $\mathbb{Z}$-SFTs are exactly the non-negative rational multiples of the logarithm of Perron numbers. (A Perron number is a real algebraic integer greater than 1 and greater than the modulus of its algebraic conjugates.) For $d \geq 2$, Hochman-Meyerovitch $[9$ proved that the entropy of a $\mathbb{Z}^{d}$-SFT is right recursively enumerable, i.e., it is the infimum of a monotonic recursive sequence of rational numbers. However, what is still lacking is the general entropy formulae of $\mathbb{Z}^{d}$-SFTs for $d \geq 2$. Some approximation algorithms for the entropies of $\mathbb{Z}^{d}$-SFTs can be found in $7,13,14$.

This paper intends to find mainly the entropy formula of $S_{d}$-SFTs. As a byproduct of our method, we also compute the entropy of the golden mean shift on $F_{d}$. For an $S_{d^{-}}$SFT $X_{\mathcal{F}}$, the number $\left|B_{n}\left(X_{\mathcal{F}}\right)\right|$ satisfies the so-called system of nonlinear recursive equations [3] (SNRE, defined in Section 2); thus, the computation of (1.1) reduces to solve its corresponding SNRE. The main difficulty in carrying out this approach is that $\left|B_{n}\left(X_{\mathcal{F}}\right)\right|$ behaves approximately like $\lambda_{1} \lambda_{2}^{\kappa^{n}}$ for some $\lambda_{1}, \lambda_{2}$, and $\left.\kappa \in \mathbb{R}(\mathrm{cf} .15,16]\right)$. That 
is,

$$
\left|B_{n}\left(X_{\mathcal{F}}\right)\right| \approx \lambda_{1} \lambda_{2}^{\kappa^{n}}
$$

the number $\ln \kappa$ is called the degree of $X_{\mathcal{F}}$ and one can derive it via the following formula $\lim _{n \rightarrow \infty} \frac{\ln ^{2}\left|B_{n}\left(X_{\mathcal{F}}\right)\right|}{n}$, where $\ln ^{2}=\ln \circ \ln$. If $\kappa=d$, we easily obtain that $h\left(X_{\mathcal{F}}\right)=\ln \lambda_{2}$. Equation 1.2 can be interpreted in the following two perspectives.

1. (symbols and generators): If $d=1$, we consider the one-sided $\mathbb{Z}$-subshifts, it is known that $\left|B_{n}\left(X_{\mathcal{F}}\right)\right| \approx \lambda_{1} \lambda_{2}^{n}$ and one can regard such formulation as that we could use $\lambda_{2}$ (in average) colors to fill up the elements of $E_{n}$ in $\mathbb{Z}$, e.g., if $X$ is a full shift with $\mathcal{A}=\{1,2\}$, then $\left|B_{n}\left(X_{\mathcal{F}}\right)\right|=2^{n}$ while $\left|E_{n}\right|=n$. Similarly, if $G=S_{d}$, the formula 1.2 represents that we could use $\lambda_{2}$ colors to fill up the elements of $E_{n}$ in $S_{d}$ (note that $\left|E_{n}\right| \approx d^{n}$ in $\left.S_{d}\right)$. Thus, it can be symbolized as $\left|B_{n}\left(X_{\mathcal{F}}\right)\right| \approx \lambda_{1} \lambda_{2}^{\kappa^{n}} \approx|\mathcal{A}|^{\left|E_{n}\right|}$. The important point to note here is that the value $\kappa$ is not always an integer. In fact, we show that $\left\{\ln \kappa: \ln \kappa\right.$ is a degree of an $\left.S_{d}-S F T\right\}=\left\{\frac{1}{p} \ln \lambda: \lambda \in \mathcal{P}, p \geq 1\right\}$, where $\mathcal{P}$ stands for the set of Perron numbers 4 ;

2. (speed): It can be easily seen that the value $\kappa$ (resp. $\lambda_{2}$ and $\lambda_{1}$ ) indicates the highest (resp. the middle and the lowest) speed of $\left|B_{n}\left(X_{\mathcal{F}}\right)\right|$ in $(1.2)$. By speed, we mean the growth rate of patterns according to the size of their supports. The more detailed information of the tuple $\left(\kappa, \lambda_{2}, \lambda_{1}\right) \in \mathbb{R}^{3}$ we know, the more exact value of $\left|B_{n}\left(X_{\mathcal{F}}\right)\right|$ we obtain.

We will restrict our attention to the entropy formulae of $S_{d^{-}}$SFTs. Due to the equivalence of the studies of $\left|B_{n}\left(X_{\mathcal{F}}\right)\right|$ and SNRE, we embark our study on analyzing all kinds of SNREs. Various types of SNREs, namely, the equal growth, dominated, oscillating, and cooperate types (write $\mathbf{E}, \mathbf{D}, \mathbf{O}, \mathbf{C}$, respectively), are presented in Section 3 , and the entropy formulae of these types are presented therein. Furthermore, we give the complete characterization of $h\left(X_{\mathcal{F}}\right)$ of $(d, k)=(2,2)$. That is, all the SNREs of $S_{2}$-SFTs with $\mathcal{A}=\{1,2\}$ are equal to $\mathbf{E} \cup \mathbf{D} \cup \mathbf{O} \cup \mathbf{C}$.

The hom-shifts, which is an important class of G-SFTs, are defined in [6] and motivated from the mathematical and statistical physics. A hom-shift can be described as a nearest neighborhood SFT with the 'symmetric' and 'isotropic' properties. That is, if $\left(a, s_{i}, b\right) \in \mathcal{F}$ for some $1 \leq i \leq d$ then $\left(a, s_{j}, b\right) \in \mathcal{F}$ for all $1 \leq j \leq d$. If $\mathcal{A}=\{1,2\}$ and $G=\mathbb{Z}^{2}, X_{\mathcal{F}}$ is the well-known two-dimensional golden mean shift ( $\mathbb{Z}^{2}$-GMS) if

$$
\mathcal{F}=\left\{\left(2, s_{i}, 2\right): i=1,2\right\}
$$

It is obvious that a $\mathbb{Z}^{2}-G M S$ is a hom-shift, and its entropy $h\left(X_{\mathcal{F}}\right)$ is called the hard square entropy constant. Either finding a closed-form for $h\left(X_{\mathcal{F}}\right)$ or determining whether this number is algebraic is still an open problem. Two important types of hom-shifts on 
$S_{d}$, the $S_{d}$-GMS and the $k$-colored chessboard on $S_{d}$, are introduced, and their entropy formulae are established in Section 4. Recently, Petersen-Salama [15] find upper and lower bounds of a hom-shift by means of the topological entropies of one-dimensional SFTs, and such result gives the relation between $S_{d}$-SFTs and $\mathbb{Z}$-SFTs. If $X_{\mathcal{F}}$ is an $F_{d}$-GMS on two symbols, i.e., $\mathcal{F}=(1.3)$, Piantadosi 16 studies the tuple $\left(\kappa, \lambda_{2}, \lambda_{1}\right) \in \mathbb{R}^{3}$ of 1.2 and shows that $\kappa=2 d-1, \lambda_{2} \approx 0.909155$. Meanwhile, the value $\lambda_{1}$ is not well-defined. It is unclear whether there is some lower-order indicator between $\lambda_{1}$ and $\lambda_{2}$. The results of $S_{d}$-SFTs developed in Section 3 can be applied to find the entropy of $F_{d}$-GMS, which provides a different approach than [16]. Some open problems on the entropy theory of $F_{d^{-}}$ or $S_{d}$-SFTs are illustrated in Section 5 .

We summary the main results of this paper as follows.

(1) Four types, namely E, D, C, O types, are introduced. Their entropy formulae are presented in Propositions 3.3, 3.6, 3.8, and 3.10 respectively. Furthermore, the set of all SNREs with $(d, k)=(2,2)$ is equal to $\mathbf{E} \cup \mathbf{D} \cup \mathbf{C} \cup \mathbf{O}$ (Section 3.5). This gives a complete characterization for the entropies of $S_{2}$-SFTs with two symbols.

(2) Two types of hom-shifts on $S_{d}$, the $S_{d}$-GMS and $k$-colored chessboard on $S_{d}$, are introduced. The entropy formulae of these two types are presented (Corollary 4.2). The entropy formula of the $F_{d}$-GMS is also developed by using the method of entropy theory on $S_{d}$-SFTs (Theorem 4.4).

\section{Preliminaries}

In this section we set up the notations of $S_{d}$-SFTs and present some known results. Some notations will be adjusted to meet the conditions of previous work in $[3$. Let $\mathcal{A}=$ $\{1, \ldots, k\}$ be the symbol set. Suppose $S_{d}$ is a free semigroup generated by $\Sigma=\{1, \ldots, d\}$. The Cayley graph of $S_{d}$ is visualized as a rooted $d$-tree. Thus, the shift space $\left(\mathcal{A}^{\left.S_{d}, \sigma\right)}\right.$ is also called a full tree-shift in [1,3]. A coloring (also call a labeled tree) in $\mathcal{A}^{S_{d}}$ is a function $f: S_{d} \rightarrow \mathcal{A}$. For $w=w_{0} w_{1} \cdots w_{n-1} \in S_{d}$ with $w_{i} \in \Sigma, \sigma_{w} f$ is defined as $\left(\sigma_{w} f\right)(g)=f(w g)$ for $g \in S_{d}$. Suppose $n \in \mathbb{N} \cup\{0\}, E_{n}=\bigcup_{k=0}^{n} \Sigma^{k}$ denotes the set of words of length at most $n$, where $\Sigma^{0}=\{\epsilon\}$ consists of the identity element of $S_{d}$. We call a function $u: E_{n} \rightarrow \mathcal{A}$ an $n$-block. The $n$-block $u$ can be written as $u=\left(u_{\epsilon} ; \sigma_{1} u, \ldots, \sigma_{d} u\right)$, where $u_{w}:=u(w)$ denotes the label of $u$ at $w \in S_{d}$, and $\sigma_{i} u$ is the $(n-1)$-block for $i=1, \ldots, d$. For instance, suppose $\mathcal{B}$ is the collection of 1 -blocks. Then every $u \in \mathcal{B}$ is written as $u=\left(i ; i_{1}, \ldots, i_{d}\right)$, where $i, i_{1}, \ldots, i_{d} \in \mathcal{A}$. Given the forbidden set $\mathcal{F}$, one can construct the associated set of admissible 1-blocks (also called basic set) as $\mathcal{B}=\bigcup_{i=1}^{k} \mathcal{B}^{(i)}$, where $\mathcal{B}^{(i)}=\left\{\left(i ; i_{1}, \ldots, i_{d}\right):\left(i, j, i_{j}\right) \notin \mathcal{F}, 1 \leq j \leq d\right\}$. Let $X^{\mathcal{B}}\left(=X_{\mathcal{F}}\right)$ be the $S_{d^{-}}$SFT constructed by $\mathcal{B}$. 
Example 2.1. Let $d=2, \mathcal{A}=\{1,2\}$ and $\mathcal{F}=\{(2, i, 2): i=1,2\}$. The corresponding basic set is $\mathcal{B}=\bigcup_{i=1}^{2} \mathcal{B}^{(i)}$, where

$$
\mathcal{B}^{(1)}=\{(1 ; 1,1),(1 ; 1,2),(1 ; 2,1),(1 ; 2,2)\} \quad \text { and } \quad \mathcal{B}^{(2)}=\{(2 ; 1,1)\}
$$

Then $X^{\mathcal{B}}=X_{\mathcal{F}}$ is the $S_{2}$-GMS.

For an $X^{\mathcal{B}}$, the system of nonlinear recursive equations is a useful tool to analyze the growth behavior of $\left|B_{n}\left(X^{\mathcal{B}}\right)\right|[3]$. Similar to the notions of monomials of multiple variables of degree $d \in \mathbb{N}$, suppose $\delta_{i ; n} \in \mathbb{R}, 1 \leq i \leq k$, are indeterminates. For $n \in \mathbb{N}$, denote by $\Delta_{n}^{d}=\left\{\delta_{1 ; n}^{i_{1}} \cdots \delta_{k ; n}^{i_{k}}: i_{1}+\cdots+i_{k}=d\right\}$ the collection of products of $\left\{\delta_{i ; n}\right\}_{i=1}^{k}$ with degree $d$.

Definition 2.2. (1) Let $F=\sum_{\mathbf{a} \in \Delta_{n}^{d}} f_{\mathbf{a}} \mathbf{a}$, where $f_{\mathbf{a}} \in \mathbb{Z}^{+}$and $\mathbf{a} \in \Delta_{n}^{d}$. The vector $v_{F}=\left(f_{\mathbf{a}}\right)_{\mathbf{a} \in \Delta_{n}^{d}}$ is called the indicator vector of $F$.

(2) A sequence $\left\{\delta_{1 ; n}, \ldots, \delta_{k ; n}\right\}_{n \in \mathbb{N}}$ is defined by a system of nonlinear recursive equation (SNRE) of degree $(d, k)$ if

$$
\delta_{i ; n}=F^{(i)}=F^{(i)}\left(\delta_{1 ; n-1}, \delta_{2 ; n-1}, \ldots, \delta_{k ; n-1}\right), \quad n \geq 2,1 \leq i \leq k
$$

and $\delta_{i ; 1}=\delta^{(i)}$ for $1 \leq i \leq k$, where $F^{(1)}, \ldots, F^{(k)}$ are linear combinations of elements of $\Delta_{n-1}^{d}$ with coefficients in $\mathbb{Z}^{+}$, respectively. We also call $F=\left\{F^{(1)}, \ldots, F^{(k)}\right\}$ an SNRE.

For $1 \leq i \leq k$ and $n \in \mathbb{N}$, let $\gamma_{i ; n}$ be the number of $n$-blocks of $X^{\mathcal{B}}$ with the root $\epsilon$ being colored by the symbol $i$. Observe that $\gamma_{i ; 0}=1$ for $1 \leq i \leq k$. The following result indicates that the number $\left|B_{n}\left(X^{\mathcal{B}}\right)\right|$ can be described by its corresponding SNRE.

Theorem 2.3 (Ban-Chang, 2017, [3] ). Given a basic set $\mathcal{B}$ and the corresponding $S_{d}-S F T$ $X^{\mathcal{B}}$. Then the values $\left\{\gamma_{i ; n}\right\}_{i=1}^{k}$ satisfy the following SNRE

$$
\begin{aligned}
\gamma_{i ; n} & =\sum_{\left(i ; i_{1}, \ldots, i_{d}\right) \in \mathcal{B}} \prod_{j=1}^{d} \gamma_{i_{j} ; n-1}, \\
\gamma_{i ; 1} & =\sum_{\left(i ; i_{1}, i_{2}, \ldots, i_{d}\right) \in \mathcal{B}} \prod_{j=1}^{d} \gamma_{i_{j} ; 0}=\left|B_{1}\left(X_{i}^{\mathcal{B}}\right)\right|, \quad 1 \leq i \leq k,
\end{aligned}
$$

where $X_{i}^{\mathcal{B}}=\left\{f \in X^{\mathcal{B}}: f(\epsilon)=i\right\}$.

Instead of reproving Theorem 2.3, we give an example below so that it is seen that the theorem holds. 
Example 2.4 (Continued). Let $X^{\mathcal{B}}$ be defined as in Example 2.1. Since $\mathcal{B}^{(1)}=\{(1 ; 1,1)$, $(1 ; 1,2),(1 ; 2,1),(1 ; 2,2)\}$, we obtain a recursive formula for $\gamma_{1 ; n}$ as follows:

$$
\gamma_{1 ; n}=\left(\gamma_{1 ; n-1}\right)^{2}+2 \gamma_{1 ; n-1} \gamma_{2 ; n-1}+\left(\gamma_{2 ; n-1}\right)^{2} .
$$

Similarly, since $\mathcal{B}^{(2)}=\{(2 ; 1,1)\}$, we have $\gamma_{2 ; n}=\left(\gamma_{1 ; n-1}\right)^{2}$. The SNRE of $X^{\mathcal{B}}$ is as follows:

$$
\begin{aligned}
& \gamma_{1 ; n}=\left(\gamma_{1 ; n-1}\right)^{2}+2 \gamma_{1 ; n-1} \gamma_{2 ; n-1}+\left(\gamma_{2 ; n-1}\right)^{2}, \\
& \gamma_{2 ; n}=\left(\gamma_{1 ; n-1}\right)^{2}, \quad \gamma_{1 ; 1}=4 \quad \text { and } \quad \gamma_{2 ; 1}=1 .
\end{aligned}
$$

Let $\Gamma=\left\{\gamma_{1}, \ldots, \gamma_{k}\right\}$ and $\Gamma_{n}=\left\{\gamma_{1 ; n}, \ldots, \gamma_{k ; n}\right\}$ for $n \geq 1$, where $\gamma_{i}$, which is the number of colorings with root colored by $i$, is introduced for the convenience of discussion. Suppose $F$ is an SNRE. For $a, b \in \Gamma$, we say that $a$ induces $b$ (or $b$ appears in $a$ ), write $a \rightarrow b$, if $b_{n-1}$ appears in some term of $a_{n}$. Precisely, $a_{n}=f_{n-1} b_{n-1}+g_{n-1}$ where $f_{n-1}$ is a nonzero polynomial on $\Gamma_{n-1}$ and $g_{n-1}$ does not contain $b_{n-1}$. We say that a connects to $b$ if there exists a path $a=c_{0}, c_{1}, c_{2}, \ldots, c_{m}=b$ such that $c_{i} \rightarrow c_{i+1}, c_{i} \in \Gamma$, for $i=0, \ldots, m-1$. Observe that, when the considered SNRE is obtained from some basic set $\mathcal{B}, a_{n} \in \mathbb{N}$ for each $a \in \Gamma$, without loss of generality, no symbol is redundant in the shift space. We say that $a$ is an essential symbol if there exists an integer $n \in \mathbb{N}$ such that $a_{n} \geq 2$ and call a inessential if it is not essential, i.e., $a_{n}=1$ for all $n \in \mathbb{N}$. We use the notation $\mathcal{E}(F)$ (resp. $\mathcal{I}(F)$ ) to denote the set of the essential symbols (resp. inessential symbols) of $F$.

Lemma 2.5. Let $F$ be an $S N R E$. If $b \in \mathcal{E}(F)$ and $a \rightarrow b$, then $a \in \mathcal{E}(F)$.

Proof. Since $b \in \mathcal{E}(F)$, there exists $m \in \mathbb{N}$ such that $b_{m-1} \geq 2$. Furthermore, $a \rightarrow b$ means that $a_{n}=f_{n-1} b_{n-1}+g_{n-1}$ for $n \in \mathbb{N}$. Thus $a_{m}=f_{m-1} b_{m-1}+g_{m-1} \geq 2$. This completes the proof.

Given an SNRE $F$, the determination of $a \in \mathcal{E}(F)$ or $a \in \mathcal{I}(F)$ can be carried out as follows. Without loss of generality, we assume that for each pair of distinct symbols $a, b \in \Gamma$, either $a$ connects to $b$ or $b$ connects to $a$. Otherwise, we decompose the set of symbols $\Gamma=\Gamma^{1} \cup \Gamma^{2} \cup \cdots \cup \Gamma^{r}$ as for each $a, b \in \Gamma^{i}$, either $a$ connects to $b$ or $b$ connects to $a$. That is, we decompose $\Gamma$ into several "irreducible" components; the discussion of "reducible" $\Gamma$ is analogous to the technique used in the classical symbolic dynamical systems. Let $\mathcal{S}_{1} \subseteq \Gamma$ be the set of $a \in \Gamma$ with $a_{1} \geq 2$. We define $\mathcal{S}_{l}$ by induction as for any $a \in \mathcal{S}_{l}$ there exists $b \in \mathcal{S}_{l-1}$ such that $a \rightarrow b$. Since $\Gamma$ is finite $(|\Gamma|=k)$, this algorithm will stop up to $k$-steps. Thus we have $\mathcal{S}=\mathcal{S}_{1} \cup \mathcal{S}_{2} \cup \cdots \cup \mathcal{S}_{q} \subseteq \Gamma$. It follows from Lemma 2.5 that $\mathcal{S} \subseteq \mathcal{E}(F)$. Let $\mathcal{R}=\Gamma \backslash \mathcal{S}$, we claim that $\mathcal{R}=\mathcal{I}(F)$. If $a \in \mathcal{R}$, this means that $a \notin \mathcal{S}_{l}$ for $l=1, \ldots, q$, thus $a_{1}=1$. Suppose there is an integer $m \in \mathbb{N}$ such that $a_{m} \geq 2$. Then $a$ must induce some $b \in \mathcal{S}$ or $a_{m}$ has at least two items. If $a_{m}$ has at least two terms, 
then $a_{1} \geq 2$, which is a contradiction. If $a$ induces some $b \in \mathcal{S}$, then $a \in \mathcal{S}$, which is also a contradiction. Thus $a_{n}=1$ for all $n \in \mathbb{N}$. That is, $a \in \mathcal{I}(F)$. On the other hand, if $a \in \mathcal{I}(F)$, it follows from Lemma 2.5 that $a$ cannot induce any $b \in \mathcal{E}(F)$. This means that $a \notin \mathcal{S}$, i.e., $a \in \mathcal{R}$. Thus $\mathcal{R}=\mathcal{I}(F)$.

\section{Topological entropy}

In this section, we introduce four types of SNREs for which we can compute their entropies explicitly. We show that for the case $(d, k)=(2,2)$, the SNRE of a $S_{d}$-SFT is of one of these types. For general $d, k$, there are more cases to study. In this circumstance, we define $a_{n}=\gamma_{1 ; n}$ and $b_{n}=\gamma_{2 ; n}$ and use $F=\left\{F^{(a)}, F^{(b)}\right\}$ to denote the associated SNRE. For example, in Example 2.4

$$
a_{n}=F^{(a)}=a_{n-1}^{2}+2 a_{n-1} b_{n-1}+b_{n-1}^{2}, \quad b_{n}=F^{(b)}=a_{n-1}^{2} .
$$

Herein, for the sake of simplification, we drop the index $n$ but $F^{(a)}$ and $F^{(b)}$ do depend on $n$.

Remark 3.1. The notation $F=\left\{F^{(a)}, F^{(b)}\right\}$ is adequate to describe the SNRE. Let $\left|F^{(*)}\right|$ be the number of terms of $F^{(*)}$ for $*=a, b$. In the Definition 2.2 of SNRE, we still need the initial conditions, i.e., $a_{1}$ and $b_{1}$, to define an SNRE. However, we see that $\left|F^{(a)}\right|=a_{1}$ and $\left|F^{(b)}\right|=b_{1}$ in 2.1 . Thus, $F=\left\{F^{(a)}, F^{(b)}\right\}$ provides all information of an SNRE.

\subsection{Equal growth type}

We say an SNRE is of the equal growth type, write type $\mathbf{E}$, if $a_{n}=b_{n}$ for all $n \geq 1$.

Proposition 3.2. Let $F$ be an SNRE associated to some $X^{\mathcal{B}}$. If $a_{1}=b_{1}$, then $a_{n}=b_{n}$ for all $n \in \mathbb{N}$.

Proof. Assume $a_{n-1}=b_{n-1}$. The fact of $a_{1}=b_{1}$ indicates that $F^{(a)}$ and $F^{(b)}$ have the same number of items (see Remark 3.1), and each item is a combination of $a_{n-1}$ and $b_{n-1}$ with degree $d$. Since $a_{n-1}=b_{n-1}$, one can compare each item of $F^{(a)}$ with $F^{(b)}$ to conclude that $a_{n}=b_{n}$. The proof is thus completed by mathematical induction.

Proposition 3.3. Let $F$ be an $S N R E$ associated to some $X^{\mathcal{B}}$ with $a_{1}=b_{1}$. Then $h\left(X^{\mathcal{B}}\right)=$ $\frac{1}{2} \ln a_{1}$.

Proof. Since $a_{n}=b_{n}$ and $\left|E_{n}\right|=2^{n+1}-1$, according to 1.1 , we have

$$
h\left(X^{\mathcal{B}}\right)=\lim _{n \rightarrow \infty} \frac{1}{2^{n+1}-1} \ln \left(a_{n}+b_{n}\right)=\lim _{n \rightarrow \infty} \frac{1}{2^{n+1}-1} \ln a_{n} .
$$


It follows from Proposition 3.2 that we rewrite $a_{n}$ as $a_{n}=a_{1} a_{n-1}^{2}$. Let $\alpha_{n}=\ln a_{n}$, we have $\alpha_{n}=2 \alpha_{n-1}+\alpha_{1}$. Iterating $\alpha_{n}$ yields $\alpha_{n}=2^{n-1} \alpha_{1} \sum_{i=0}^{n-1} 2^{-i}=2^{n} \alpha_{1}\left(1-(1 / 2)^{n}\right)$. Thus

$$
h\left(X^{\mathcal{B}}\right)=\lim _{n \rightarrow \infty} \frac{2^{n} \alpha_{1}\left(1-(1 / 2)^{n}\right)}{2^{n+1}-1}=\frac{1}{2} \alpha_{1}=\frac{1}{2} \ln a_{1} .
$$

This completes the proof.

\subsection{Dominated type}

Let $F$ be an SNRE, $a \in \mathcal{A}$ is called a dominating element if $a_{n} \geq b_{n}$ for all $b \in \mathcal{A}$ and $n \in \mathbb{N}$. If $F$ admits a dominating element, we call $F$ is of the dominated type (type $\mathbf{D}$ ).

Example 3.4. Suppose $F$ is defined as

$$
a_{n}=a_{n-1}^{2}+2 a_{n-1} b_{n-1}, \quad b_{n}=a_{n-1}^{2}+a_{n-1} b_{n-1}, \quad a_{1}=3, \quad b_{1}=2 .
$$

Since $a_{1} \geq b_{1}$ and if we assume $a_{n-1} \geq b_{n-1}$, we have $a_{n} \geq b_{n}$ according to $F$. Thus $a$ is a dominating element and $F$ is of the type $\mathbf{D}$.

Lemma 3.5. Let $F$ be an SNRE associated to some $X^{\mathcal{B}}$. If $a \in \mathcal{A}$ is a dominating element, then $h\left(X^{\mathcal{B}}\right)=\lim _{n \rightarrow \infty} \frac{\ln a_{n}}{2^{n+1}-1}$.

Proof. If $a$ is a dominating element, then $1<1+\frac{b_{n}}{a_{n}} \leq 2$. So $h\left(X^{\mathcal{B}}\right)=\lim _{n \rightarrow \infty} \frac{\ln a_{n}\left(1+\frac{b_{n}}{a_{n}}\right)}{2^{n+1}-1}=$ $\lim _{n \rightarrow \infty} \frac{\ln a_{n}}{2^{n+1}-1}$. This completes the proof.

Let $\mathcal{M}_{m}$ be the collection of $m \times m$ square matrices. Suppose $F=\left\{F^{(a)}, F^{(b)}\right\}$ admits a dominating element, say $a$, we could arrange terms of $F^{(a)}$ and $F^{(b)}$ with respect to the power of $a_{n-1}$ descendingly. Let $F_{1}^{(*)}$ be the first item of $F^{(*)}$ for $*=a$ or $b$. For $*=a$ or $b$, we have

$$
F^{(*)}=c_{1} F_{1}^{(*)}+c_{2} F_{2}^{(*)}+\cdots+c_{k_{*}} F_{k_{*}}^{(*)}=F_{1}^{(*)}\left(c_{1}+\frac{c_{2} F_{2}^{(*)}}{F_{1}^{(*)}}+\cdots+\frac{c_{k_{*}} F_{k_{*}}^{(*)}}{F_{1}^{(*)}}\right) .
$$

Let

$$
r_{n-1}^{(*)}=\left(c_{1}+\frac{c_{2} F_{2}^{(*)}}{F_{1}^{(*)}}+\cdots+\frac{c_{k_{*}} F_{k_{*}}^{(*)}}{F_{1}^{(*)}}\right) .
$$

Since $F_{1}^{(*)} \geq F_{j}^{(*)}$ for all $j=2, \ldots, k_{*}$, we have

$$
1 \leq r_{n-1}^{(*)} \leq c_{1}+\cdots+c_{k_{*}} \leq 4
$$

where the number 4 comes from the extreme case where $c_{1}=c_{3}=1$ and $c_{2}=2$. Let $\alpha_{n}=\ln a_{n}$ and $\beta_{n}=\ln b_{n}$ and $v_{n}=\left(\begin{array}{c}\alpha_{n} \\ \beta_{n}\end{array}\right)$. Combining these with (3.1), we deduce

$$
v_{n}=K v_{n-1}+\ln r_{n-1}, \quad \text { where } \ln r_{n-1}:=\left(\begin{array}{c}
\ln r_{n-1}^{(a)} \\
\ln r_{n-1}^{(b)}
\end{array}\right),
$$


and $K \in \mathcal{M}_{2}$. Denote by $v^{[i]}$ the $i$-th element of a vector $v$ and $R=\left(\begin{array}{cc}1 & 1 \\ 1 & -1\end{array}\right)$ so that $\left(\begin{array}{ll}1 & 1 \\ 1 & 1\end{array}\right)=R\left(\begin{array}{ll}2 & 0 \\ 0 & 0\end{array}\right) R^{-1}$. Note that $\left(\ln r_{n-1}\right)^{[1]}=\ln r_{n-1}^{(a)}$ and $\left(\ln r_{n-1}\right)^{[2]}=\ln r_{n-1}^{(b)}$.

Proposition 3.6. Let $F$ be an SNRE associated to some $X^{\mathcal{B}}$ which admits a dominating element $a \in \mathcal{A} \cap \mathcal{E}(F)$. Then $F_{1}^{(a)} \neq b_{n-1}^{2}$, and there are the following three cases.

(1) $F_{1}^{(a)}=a_{n-1}^{2}$, then

$$
h\left(X^{\mathcal{B}}\right)=\frac{1}{4} \lim _{n \rightarrow \infty}\left(\ln a_{1}+\sum_{j=1}^{n-1} 2^{-j} \ln r_{j}^{(a)}\right)
$$

(2) $F_{1}^{(a)}=a_{n-1} b_{n-1}$ and $F_{1}^{(b)}=a_{n-1} b_{n-1}$, then

$$
h\left(X^{\mathcal{B}}\right)=\frac{1}{4} \lim _{n \rightarrow \infty}\left(\left(\widehat{v}_{1}\right)^{[1]}+\sum_{j=1}^{n-1} 2^{-j}\left(\ln \widehat{r}_{j}\right)^{[1]}\right),
$$

where $\widehat{v}_{1}=R^{-1} v_{1}$ and $\ln \widehat{r}_{j}=R^{-1} \ln r_{j}$;

(3) $F_{1}^{(a)}=a_{n-1} b_{n-1}$ and $F_{1}^{(b)}=b_{n-1}^{2}$, then the number $\left|B_{n}\left(X^{\mathcal{B}}\right)\right|$ is up to exponential. In this case, we have $h\left(X^{\mathcal{B}}\right)=0$.

Proof. Since $a$ is a dominating element, i.e., $a_{n} \geq b_{n}$, the equality $F_{1}^{(a)}=b_{n-1}^{2}$ implies that $F_{1}^{(b)}=b_{n-1}^{2}$ and so $b_{n}=1$ for all $n$, i.e., $b \in \mathcal{I}(F)$. Consequently, $F_{1}^{(a)}=b_{n-1}^{2}=1$; that is, $a \in \mathcal{I}(F)$, which is a contradiction. Hence $F_{1}^{(a)} \neq b_{n-1}^{2}$. Thus, there are two possibilities for $F_{1}^{(a)}$; namely, $F_{1}^{(a)}=a_{n-1}^{2}$ or $F_{1}^{(a)}=a_{n-1} b_{n-1}$.

(i) $F_{1}^{(a)}=F_{1}^{(b)}=a_{n-1}^{2}$. It follows from the preceding algorithm that we have $K=$ $\left(\begin{array}{ll}2 & 0 \\ 2 & 0\end{array}\right)$. Iterating the recursive formula (3.3) we obtain $v_{n}=K^{n-1} v_{1}+\sum_{j=1}^{n-1} K^{n-j-1} \ln r_{j}$. Combining this with the fact that $K^{n}=\left(\begin{array}{cc}2^{n} & 0 \\ 2^{n} & 0\end{array}\right)$ we have

$$
\begin{aligned}
\alpha_{n} & =\ln a_{n}=\left(K^{n-1} v_{1}\right)^{[1]}+\sum_{j=1}^{n-1}\left(K^{n-j-1} \ln r_{j}\right)^{[1]} \\
& =2^{n-1} \ln a_{1}+\sum_{j=1}^{n-1} 2^{n-j-1} \ln r_{j}^{(a)} \\
& =2^{n-1}\left(\ln a_{1}+\sum_{j=1}^{n-1} 2^{-j} \ln r_{j}^{(a)}\right) .
\end{aligned}
$$

Let $A_{n}=\ln a_{1}+\sum_{j=1}^{n-1} 2^{-j} \ln r_{j}^{(a)}$. Since $1 \leq r_{j}^{(a)} \leq 4$ for all $j$ (see 3.2$)$ ), $A_{n}$ converges. Set $A_{\infty}:=\lim _{n \rightarrow \infty} A_{n}$. It follows from Lemma 3.5 that

$$
h\left(X^{\mathcal{B}}\right)=\lim _{n \rightarrow \infty} \frac{\alpha_{n}}{2^{n+1}-1}=\frac{1}{4}\left(\ln a_{1}+\sum_{j=1}^{\infty} 2^{-j} \ln r_{j}^{(a)}\right)=\frac{A_{\infty}}{4} .
$$


(ii) $F_{1}^{(a)}=a_{n-1}^{2}, F_{1}^{(b)}=a_{n-1} b_{n-1}$ or $F_{1}^{(b)}=b_{n-1}^{2}$. If $F_{1}^{(b)}=a_{n-1} b_{n-1}$, we see that $K=\left(\begin{array}{ll}2 & 0 \\ 1 & 1\end{array}\right)$. Since $K^{n}=\left(\begin{array}{cc}2^{n}-1 & 0 \\ 2^{n}-1 & 1\end{array}\right)$, identical argument is applied to show that $h\left(X^{\mathcal{B}}\right)=\frac{A_{\infty}}{4}$. Similarly, in the case where $F_{1}^{(b)}=b_{n-1}^{2}$, we have $h\left(X^{\mathcal{B}}\right)=\frac{A_{\infty}}{4}$.

(iii) $F_{1}^{(a)}=a_{n-1} b_{n-1}$ and $F_{1}^{(b)}=a_{n-1} b_{n-1}$. We see that $K=\left(\begin{array}{ll}1 & 1 \\ 1 & 1\end{array}\right)$. Let $R \in \mathcal{M}_{2}$ be defined as above. By a similar argument, we have $v_{n}=R\left(D^{n-1} R^{-1} v_{1}+\sum_{j=1}^{n-1} D^{n-j-1} R^{-1}\right.$ $\left.\ln r_{j}\right)$, where $D=\left(\begin{array}{ll}2 & 0 \\ 0 & 0\end{array}\right)$. Let $R^{-1} v_{1}=\widehat{v}_{1}$ and $R^{-1} \ln r_{j}=\ln \widehat{r}_{j}$. Then we have $v_{n}=$ $R\left(D^{n-1} \widehat{v}_{1}+\sum_{j=1}^{n-1} D^{n-j-1} \ln \widehat{r}_{j}\right)$ and

$$
\begin{aligned}
\ln a_{n} & =v_{n}^{[1]}=R_{11}\left(2^{n-1} \widehat{v}_{1}^{[1]}+\sum_{j=1}^{n-1} 2^{n-j-1}\left(\ln \widehat{r}_{j}\right)^{[1]}\right)+R_{12}\left(\ln \widehat{r}_{n-1}\right)^{[2]} \\
& =2^{n-1}\left[R_{11}\left(\widehat{v}_{1}^{[1]}+\sum_{j=1}^{n-1} 2^{-j}\left(\ln \widehat{r}_{j}\right)^{[1]}\right)\right]+R_{12}\left(\ln \widehat{r}_{n-1}\right)^{[2]} .
\end{aligned}
$$

Since $R_{11}=1, h\left(X^{\mathcal{B}}\right)=\frac{1}{4} \lim _{n \rightarrow \infty}\left(\widehat{v}_{1}^{[1]}+\sum_{j=1}^{n-1} 2^{-j}\left(\ln \widehat{r}_{j}\right)^{[1]}\right)$.

(iv) $F_{1}^{(a)}=a_{n-1} b_{n-1}$ and $F_{1}^{(b)}=b_{n-1}^{2}$. In this case one can check that $b \in \mathcal{I}(F)$, $K=\left(\begin{array}{ll}1 & 1 \\ 0 & 2\end{array}\right)$ and $K^{n}=\left(\begin{array}{cc}1 & 2^{n}-1 \\ 0 & 2^{n}\end{array}\right)$. Since $a \in \mathcal{E}(F)$ and $\left(\ln r_{j}\right)^{[2]}=0\left(b_{n}=1\right)$, we have

$$
\begin{aligned}
\alpha_{n} & =v_{n}^{[1]}=\left(K^{n-1} v_{1}\right)^{[1]}+\sum_{j=1}^{n-1}\left(K^{n-j-1} \ln r_{j}\right)^{[1]} \\
& =\ln a_{1}+\sum_{j=1}^{n-1}\left(\ln r_{j}\right)^{[1]}=\ln a_{1}+\sum_{j=1}^{n-1} \ln r_{j}^{(a)} \leq c n
\end{aligned}
$$

for some constant $c$. Thus, the growth rate of $a_{n}$ is up to exponential. Meanwhile, $h\left(X^{\mathcal{B}}\right)=0$. This completes the proof.

Example 3.7. Let $F=\left\{F^{(a)}, F^{(b)}\right\}$ be defined as

$$
a_{n}=2 a_{n-1} b_{n-1}, \quad b_{n}=b_{n-1}^{2}, \quad a_{1}=2, \quad b_{1}=1 .
$$

We have $v_{n}=K v_{n-1}+\ln r_{n-1}$, where $K=\left(\begin{array}{ll}1 & 1 \\ 0 & 2\end{array}\right)$ and $\ln r_{n}=\left(\begin{array}{c}\ln 2 \\ 0\end{array}\right)$ for $n \in \mathbb{N}$. Thus $v_{n}=K^{n-1} v_{1}+K^{n-2} \ln r_{1}+\cdots+\ln r_{n-1}$. Since $K^{n}=\left(\begin{array}{cc}1 & 2^{n}-1 \\ 0 & 2^{n}\end{array}\right)$, we have $\alpha_{n}=\alpha_{1}+\ln 2+$ $\cdots+\ln 2=n \ln 2$. Then, $\left(a_{n}, b_{n}\right)=\left(2^{n}, 1\right)$ for $n \geq 1$ due to the fact that $b \in \mathcal{I}(F)$.

\subsection{Cooperating type}

Let $F$ be an SNRE, and define $c_{n}=a_{n}+b_{n}$. We say that $F$ is of the cooperating type (type $\mathbf{C}$ ) if $c_{n}=c_{n-1}^{2}+g_{n-1}$, where $g_{n} \leq c_{n}^{2}$. For example, if $F$ is

$$
a_{n}=2 a_{n-1} b_{n-1}+b_{n-1}^{2}, \quad b_{n}=a_{n-1}^{2}+a_{n-1} b_{n-1}, \quad a_{1}=3, \quad b_{1}=2 .
$$

Then we have $\left(a_{n}+b_{n}\right)=\left(a_{n-1}+b_{n-1}\right)^{2}+a_{n-1} b_{n-1}$ and $a_{n-1} b_{n-1} \leq\left(a_{n-1}+b_{n-1}\right)^{2}$. 
Proposition 3.8. Let $X^{\mathcal{B}}$ be an $S_{d}$-SFT and $F$ be its SNRE. If $F$ is of the cooperating type, then

$$
h\left(X^{\mathcal{B}}\right)=\frac{1}{4}\left[\ln c_{1}+\lim _{n \rightarrow \infty} \sum_{j=1}^{n-1} 2^{-j} \ln \left(1+\frac{g_{j}}{c_{j}^{2}}\right)\right] .
$$

Proof. Let $c_{n}=a_{n}+b_{n}$. Since the corresponding SNRE $F$ is of type $\mathbf{C}$, it is seen that the symbol $c$ can be treated as a dominating element just like the case where $F$ is of type $\mathbf{D}$. The proof is almost identical to the proof of Proposition 3.6, thus we omit it.

Example 3.9. Let $F$ be defined in (3.5). We have $c_{n}=c_{n-1}^{2}+g_{n-1}$, where $g_{n}=a_{n} b_{n}$. Since $\frac{g_{n}}{c_{n}^{2}} \leq 1$, we have

$$
\theta_{n}=2^{n-1}\left(\theta_{1}+\sum_{j=1}^{n-1} 2^{-j} \ln \left(1+\frac{g_{j}}{c_{j}^{2}}\right)\right)
$$

where $\theta_{n}=\ln c_{n}$. Then $h\left(X^{\mathcal{B}}\right)=\frac{1}{4} A_{\infty}$, where

$$
A_{\infty}=\lim _{n \rightarrow \infty}\left(\theta_{1}+\sum_{j=1}^{n-1} 2^{-j} \ln \left(1+\frac{g_{j}}{c_{j}^{2}}\right)\right) .
$$

\subsection{Oscillating type}

We say that $F$ is of the oscillating type (type $\mathbf{O}$ ) if there exist two subsequences of $\mathbb{N}$, say $\left\{n_{m}^{(a)}\right\}$ and $\left\{n_{m}^{(b)}\right\}$ such that $a_{n} \geq b_{n}$ on $\left\{n_{m}^{(a)}\right\}$ and $a_{n}<b_{n}$ on $\left\{n_{m}^{(b)}\right\}$. For the case $d=k=2$, observe that the corresponding SNRE $F$ of $X^{\mathcal{B}}$ is of type $\mathbf{O}$ if and only if $\mathcal{B}=\{(1 ; 1,2),(1 ; 2,2),(2 ; 1,1)\}$ or $\mathcal{B}=\{(2 ; 1,1),(2 ; 2,1),(1 ; 2,2)\}$. In this case, the topological entropy of $X^{\mathcal{B}}$ is characterized as follows.

Proposition 3.10. Let $\mathcal{B}=\{(1 ; 1,2),(1 ; 2,2),(2 ; 1,1)\}$ be a basic set. Suppose $X^{\mathcal{B}}$ and $F$ are the corresponding $S_{2}-S F T$ and SNRE respectively. Then $F$ is of the oscillating type, and

$$
h\left(X^{\mathcal{B}}\right)=\frac{1}{4} \lim _{n \rightarrow \infty}\left(\alpha_{1}+\sum_{j=1}^{n} 2^{-2 j} \ln r_{2 j-1}^{(a)}\right),
$$

where $r_{i}^{(a)}=1+\frac{b_{i}}{a_{i}}+\left(\frac{b_{i}}{a_{i}}\right)^{2}$ and $r_{i}^{(b)}=1+2 \frac{b_{i}}{a_{i}}+\left(\frac{b_{i}}{a_{i}}\right)^{2}$.

Proof. Note that the SNRE $F$ is of the following form

$$
a_{n}=a_{n-1} b_{n-1}+b_{n-1}^{2}, \quad b_{n}=a_{n-1}^{2}, \quad a_{1}=2, \quad b_{1}=1 .
$$

Let $\tau_{n}=\frac{a_{n}}{b_{n}}$, it can be easily checked that $\tau_{n}=\frac{1}{\tau_{n-1}}+\frac{1}{\tau_{n-1}^{2}}$. Note that $\tau_{1}=2$ and $\tau_{2}=\frac{3}{4}$. We claim that $\tau_{n} \geq 2$ implies $\tau_{n+1} \leq \frac{3}{4}$ and $\tau_{n} \leq \frac{3}{4}$ infers $\tau_{n+1} \geq 2$. Indeed, if $\tau_{n} \geq 2$ then 
$\tau_{n+1}=\frac{1}{\tau_{n}}+\left(\frac{1}{\tau_{n}}\right)^{2} \leq \frac{1}{2}+\left(\frac{1}{2}\right)^{2}=\frac{3}{4}$. Whenever $\tau_{n} \leq \frac{3}{4}$, then $\tau_{n+1} \geq \frac{4}{3}+\left(\frac{4}{3}\right)^{2}=\frac{28}{9} \geq 2$. Thus we have $\gamma_{2 n+1}>1$ and $\gamma_{2 n}<1$. This means $a_{n}>b_{n}$ if $n$ is odd and $a_{n}<b_{n}$ if $n$ is even. That is, $F$ is of the oscillating type. In this case, there is no dominating element. However, if $n$ is odd, $a_{n}$ is still a dominating element, and $b_{n}$ is a dominating element for $n$ being even. Expand $F$ to the $(n-2)$ order and arrange $F^{(a)}$ and $F^{(b)}$ as follows:

$$
a_{n+2}=a_{n}^{4}+a_{n}^{3} b_{n}+a_{n}^{2} b_{n}^{2}, \quad b_{n+2}=a_{n}^{2} b_{n}^{2}+2 a_{n} b_{n}^{3}+b_{n}^{4} .
$$

Recall that $\alpha_{n}=\ln a_{n}, \beta_{n}=\ln b_{n}$, and $v_{n}=\left(\begin{array}{c}\alpha_{n} \\ \beta_{n}\end{array}\right)$. We have $v_{n+2}=P v_{n}+\ln r_{n}$, where $P=\left(\begin{array}{cc}2 & 0 \\ 2 & 2\end{array}\right)$, and

$$
\ln r_{n}=\left(\begin{array}{c}
\ln \left(1+\frac{b_{n}}{a_{n}}+\left(\frac{b_{n}}{a_{n}}\right)^{2}\right) \\
\ln \left(1+2 \frac{b_{n}}{a_{n}}+\left(\frac{b_{n}}{a_{n}}\right)^{2}\right)
\end{array}\right)=\left(\begin{array}{c}
\ln r_{n}^{(a)} \\
\ln r_{n}^{(b)}
\end{array}\right) .
$$

Iterating $v_{n+2}$ we obtain that $v_{2 n+1}=P^{n} v_{1}+\sum_{j=1}^{n} P^{n-j} \ln r_{2 j-1}$. Since $P^{n}=\left(\begin{array}{cc}2^{2 n} & 0 \\ 2^{2 n}-2^{n} & 2^{n}\end{array}\right)$, we have

$$
\alpha_{2 n+1}=2^{2 n}\left(\alpha_{1}+\sum_{j=1}^{n} 2^{-2 j} \ln r_{2 j-1}^{(a)}\right) .
$$

Set $A_{n}=\alpha_{1}+\sum_{j=1}^{n} 2^{-2 j} \ln r_{2 j-1}^{(a)}$, then $\lim _{n \rightarrow \infty} A_{n}$ exists and define $A_{\infty}=\lim _{n \rightarrow \infty} A_{n}$. Note that $\left|E_{2 n+1}\right|=\sum_{j=0}^{2 n+1} 2^{j}=2^{2 n+2}-1$. Since $a$ is a dominating element for odd $n$, combining Lemma 3.5. Proposition 3.6 with $(3.6)$ yields

$$
\lim _{n \rightarrow \infty} \frac{\ln \left(a_{2 n+1}+b_{2 n+1}\right)}{\left|E_{2 n+1}\right|}=\frac{1}{4} \lim _{n \rightarrow \infty}\left(\alpha_{1}+\sum_{j=1}^{n} 2^{-2 j} \ln r_{2 j-1}^{(a)}\right)=\frac{A_{\infty}}{4} .
$$

Since $a_{n}>b_{n}$ if $n$ is odd, $\ln r_{2 j-1}^{(a)}=\ln \left(1+\frac{b_{n}}{a_{n}}+\left(\frac{b_{n}}{a_{n}}\right)^{2}\right)<\ln 3$ for $j \in \mathbb{N}$. Hence, the limit of $A_{n}$ exists. Combining (3.7) with the fact that the limit (1.1) exists, we have $h\left(X^{\mathcal{B}}\right)=\lim _{n \rightarrow \infty} \frac{\ln \left(a_{2 n+1}+b_{2 n+1}\right)}{\left|E_{2 n+1}\right|}=\frac{A_{\infty}^{(a)}}{4}$. This completes the proof.

\subsection{Cases investigation}

Recall that $\left|F^{(*)}\right|$ denotes the number of items of $F^{(*)}$ for $*=a$ or $b$.

Lemma 3.11. Let $F$ be such that

$$
a_{n}=2 a_{n-1} b_{n-1}, \quad b_{n}=a_{n-1}^{2}, \quad a_{1}=2, \quad b_{1}=1 .
$$

Then $F$ is of the dominated type.

Proof. Let $\tau_{n}=\frac{a_{n}}{b_{n}}$. Then we have $\tau_{n}=2 \frac{b_{n-1}}{a_{n-1}}=2 \frac{1}{\tau_{n-1}}$, i.e., $\tau_{n} \tau_{n-1}=2$. Since $\tau_{1}=2$ and $\tau_{2}=1$, it can be easily checked that $\tau_{2 n-1}=2$ and $\tau_{2 n}=1$. This shows that $F$ is of the dominated type. 
Suppose $\mathcal{B} \subseteq \mathcal{A}^{E_{1}}$ and $F$ is the corresponding SNRE. It is seen that $F$ is either of type $\mathbf{D}$ or of type $\mathbf{O}$. Nonetheless, an SNRE either of type $\mathbf{E}$ or of type $\mathbf{C}$ is easier to be verified. In the following, we elaborate the type of their SNREs for some sets of basic patterns. Let $(d, k)=(2,2)$ and $X^{\mathcal{B}}$ be an $S_{d^{-}}$SFT for some $\mathcal{B} \subseteq \mathcal{A}^{E_{1}}$. Suppose $F$ is its SNRE, then $F$ is either one of the following four types.

(1) $F$ is of the equal growth type,

(2) $F$ is of the dominated type,
(3) $F$ is of the oscillating type,

(4) $F$ is of the cooperating type.

Recall that $\left|F^{(*)}\right|$ is the number of items in $F^{(*)}$. Without loss of generality, we only discuss the case where $\left|F^{(a)}\right|>\left|F^{(b)}\right|$, the case where $\left|F^{(a)}\right|<\left|F^{(b)}\right|$ is similar. For the case where $\left|F^{(a)}\right|=\left|F^{(b)}\right|$, Proposition 3.2 is applied to show it is of type $\mathbf{E}$. Thus we divide the discussion into the following small cases. (i) $\left|F^{(a)}\right|=4$. In this case, we know that $a_{n} \geq b_{n}$, thus $a$ is a dominating element. Thus $F$ is of the type $\mathbf{D}$; (ii) $\left|F^{(a)}\right|=3$. We only discuss the following subcases (a) $\left|F^{(b)}\right|=1$. Since $\left|F^{(a)}\right|=3$ there are only three possibilities: (1) $a_{n}=a_{n-1}^{2}+2 a_{n-1} b_{n-1}$; (2) $a_{n}=2 a_{n-1} b_{n-1}+b_{n-1}^{2}$; (3) $a_{n}=a_{n-1}^{2}+a_{n-1} b_{n-1}+b_{n-1}^{2}$. In case (3), since $\left|F^{(b)}\right|=1$, we conclude that $a$ is a dominating element. Thus $F$ is of the type $\mathbf{D}$. In case (2), if $b_{n}=a_{n-1} b_{n-1}$ or $b_{n-1}^{2}$, then $a$ is also a dominating element and $F$ is of the type $\mathbf{D}$. Thus we only need to discuss $b_{n}=a_{n-1}^{2}$. That is,

$$
F_{I}: a_{n}=2 a_{n-1} b_{n-1}+b_{n-1}^{2}, \quad b_{n}=a_{n-1}^{2}, \quad a_{1}=3, \quad b_{1}=1 .
$$

It can be easily checked that $F_{I}$ is of the type $\mathbf{C}$. For case (1), if $b_{n}=a_{n-1}^{2}$ or $a_{n-1} b_{n-1}$, then $a$ is a dominating element and $F$ is of the type $\mathbf{D}$, thus we only need to discuss $b_{n}=b_{n-1}^{2}$. However, in this case $b_{n}=1$, i.e., $b \in \mathcal{I}(F)$. Thus $a$ is still a dominating element, i.e., $F$ is of the type D. (b) $\left|F^{(b)}\right|=2$. It follows the same argument as case (a), we also has three cases (1)-(3) and $b_{n}$ has the following cases: $b_{n}=a_{n-1}^{2}+a_{n-1} b_{n-1}$ or $2 a_{n-1} b_{n-1}$ or $a_{n-1} b_{n-1}+b_{n-1}^{2}$ or $a_{n-1}^{2}+b_{n-1}^{2}$. Under the same arguments as above, we only need to discuss the following cases:

$$
\begin{array}{rlll}
F_{I I}: & a_{n}=2 a_{n-1} b_{n-1}+b_{n-1}^{2}, \quad b_{n}=a_{n-1}^{2}+a_{n-1} b_{n-1}, & a_{1}=3, & b_{1}=2, \\
F_{I I I}: & a_{n}=a_{n-1}^{2}+a_{n-1} b_{n-1}+b_{n-1}^{2}, \quad b_{n}=2 a_{n-1} b_{n-1}, & a_{1}=3, & b_{1}=2, \\
F_{I V}: & a_{n}=a_{n-1}^{2}+2 a_{n-1} b_{n-1}, \quad b_{n}=a_{n-1} b_{n-1}+b_{n-1}^{2}, & a_{1}=3, & b_{1}=2 .
\end{array}
$$

For $F_{I I}$, we see that $\left(a_{n}+b_{n}\right)=\left(a_{n-1}+b_{n-1}\right)^{2}+a_{n-1} b_{n-1}$, thus $F$ is of the type $\mathbf{C}$. For $F_{I I I}$, let $\tau_{n}=\frac{a_{n}}{b_{n}}$, then we have $\tau_{n}=\frac{1}{2}+\frac{\tau_{n-1}}{2}+\frac{1}{2 \tau_{n-1}}$. Since $\tau_{1}=\frac{3}{2}>1$, we conclude that $\tau_{n}>1$ for all $n \geq 1$ by induction, i.e., $a$ is a dominating element and $F_{I I I}$ is of the type $\mathbf{D}$. The discussion of $F_{I V}$ is the same as $F_{I I}$; that is, $F_{I V}$ is of the type $\mathbf{C}$; (iii) $\left|F^{(a)}\right|=2$. There are only four possibilities: (1) $a_{n}=a_{n-1}^{2}+a_{n-1} b_{n-1}$; (2) $a_{n}=2 a_{n-1} b_{n-1}$; (3) $a_{n}=$ 
$a_{n-1} b_{n-1}+b_{n-1}^{2}$ and (4) $a_{n}=a_{n-1}^{2}+b_{n-1}^{2}$. Since $\left|F^{(a)}\right|>\left|F^{(b)}\right|$, we only need to discuss the case where $\left|F^{(b)}\right|=1$. That is, $b_{n}=a_{n-1}^{2}$ or $a_{n-1} b_{n-1}$ or $b_{n-1}^{2}$. If $b_{n}=b_{n-1}^{2}$, then $b \in \mathcal{I}(F)$ and $a$ is a dominating element; this means $F$ is of the type $\mathbf{D}$. Thus we only have the following three cases:

$$
\begin{aligned}
F_{V}: & a_{n}=2 a_{n-1} b_{n-1}, \quad b_{n}=a_{n-1}^{2}, \quad a_{1}=2, \quad b_{1}=1, \\
F_{V I}: & a_{n}=a_{n-1} b_{n-1}+b_{n-1}^{2}, \quad b_{n}=a_{n-1}^{2}, \quad a_{1}=2, \quad b_{1}=1, \\
F_{V I I}: & a_{n}=a_{n-1}^{2}+b_{n-1}^{2}, \quad b_{n}=a_{n-1} b_{n-1}, \quad a_{1}=2, \quad b_{1}=1 .
\end{aligned}
$$

For case $F_{V}$, Lemma 3.11 shows that it is of the type $\mathbf{D}$. The proof of Proposition 3.10 indicates that $F_{V I}$ is of the type $\mathbf{O}$. For case $F_{V I I}$, we assume $\tau_{n}=\frac{a_{n}}{b_{n}}=\tau_{n-1}+\frac{1}{\tau_{n-1}}$. Since $\tau_{1} \geq 2$, it can be checked that $\tau_{n} \geq 2$ for all $n$ by induction. Thus $a$ is a dominating element and $F_{V I I}$ is of the type $\mathbf{D}$.

\subsection{Numerical results}

Recall that $v_{F}=\left(f_{\mathbf{a}}\right)_{\mathbf{a} \in \mathcal{A}^{d}}$ is the indicator vector of $F$ (Definition 2.2). For $k=2$ we denote by $v^{(a)}=v_{F^{(a)}}$ and $v^{(b)}=v_{F^{(b)}}$. In this subsection, we provide some numerical results for the entropies of the case where $(d, k)=(2,2)$. The case where $\left|F^{(a)}\right|=\left|F^{(b)}\right|$ immediately follows from the Proposition 3.3 , thus we omit it. The following tables are the entropies of the cases where $\left|F^{(a)}\right|>\left|F^{(b)}\right|$ and $\left|F^{(a)}\right|=2,3$, and 4, respectively.

\begin{tabular}{|c|c|c|c|c|}
\hline$v^{(b)} \backslash v^{(a)}$ & $(1,1,0)$ & $(1,0,1)$ & $(0,1,1)$ & $(0,2,0)$ \\
\hline$(1,0,0)$ & 0.285443 & 0.254262 & 0.214332 & 0.346235 \\
\hline$(0,1,0)$ & 0.253877 & 0.216424 & 0.252677 & 0.295580 \\
\hline$(0,0,1)$ & 0.234348 & 0.203677 & 0 & 0 \\
\hline
\end{tabular}

\begin{tabular}{|c|c|c|c|}
\hline$v^{(b)} \backslash v^{(a)}$ & $(1,1,1)$ & $(1,2,0)$ & $(0,2,1)$ \\
\hline$(1,0,0)$ & 0.404347 & 0.429271 & 0.517933 \\
\hline$(0,1,0)$ & 0.346538 & 0.372742 & 0.427385 \\
\hline$(0,0,1)$ & 0.325765 & 0.346574 & 0 \\
\hline$(1,1,0)$ & 0.474630 & 0.490218 & 0.527259 \\
\hline$(1,0,1)$ & 0.462992 & 0.480426 & 0.523983 \\
\hline$(0,1,1)$ & 0.432619 & 0.451472 & 0.516799 \\
\hline$(0,2,0)$ & 0.455134 & 0.472200 & 0.522268 \\
\hline
\end{tabular}

\begin{tabular}{|c|c|}
\hline$v^{(b)} \backslash v^{(a)}$ & $(1,2,1)$ \\
\hline$(1,0,0)$ & 0.508156 \\
\hline$(0,1,0)$ & 0.432802 \\
\hline$(0,0,1)$ & 0.407355 \\
\hline$(1,1,0)$ & 0.570417 \\
\hline$(1,0,1)$ & 0.556489 \\
\hline$(0,1,1)$ & 0.507662 \\
\hline$(0,2,0)$ & 0.537203 \\
\hline$(1,1,1)$ & 0.625995 \\
\hline$(1,2,0)$ & 0.633417 \\
\hline$(0,2,1)$ & 0.611294 \\
\hline
\end{tabular}




\section{Hom-shifts}

\subsection{Golden mean shifts and $k$-colored chessboard}

Let $k \geq 2$ and $\mathcal{A}$ be the set of symbols such that $|\mathcal{A}|=k$. Suppose $\mathbb{A}=\left(A_{i}\right)_{i=1}^{d} \in \mathcal{M}_{k}^{d}$ is the $d$-tuple of the $k \times k$ binary matrices such that $A_{i}$ is indexed by $\mathcal{A}$. The $S_{d}$-vertex shift $X_{\mathbb{A}}$ is defined by $\mathbb{A}$ in which $A_{i}(a, b)=0$ if and only if $\left(a, s_{i}, b\right) \in \mathcal{F}$. That is

$$
X_{\mathbb{A}}=\left\{x \in \mathcal{A}^{S_{d}}: A_{i}\left(x(g), x\left(g s_{i}\right)\right)=1 \text { for } g \in S_{d}, 1 \leq i \leq d\right\} .
$$

In [2], we prove that an $S_{d^{-}}$SFT is conjugate to an $S_{d^{-}}$-vertex shift and vice versa. We call an $S_{d}$-vertex shift $X=X_{\mathbb{A}}$ hom-shift if $A_{i}=A \in \mathcal{M}_{k}$ for all $i$, and we write $X_{\mathbb{A}}=X_{A}$ if it causes no confusion. $X_{\mathbb{A}}$ is called a golden mean shift if $X$ is a hom-shift and $A$ is of the following form

$$
A(i, j)= \begin{cases}0 & \text { if } i=j=k \\ 1 & \text { otherwise }\end{cases}
$$

A hom-shift $X_{A}$ is called $k$-colored chessboard if

$$
A(i, j)= \begin{cases}0 & \text { if } i=j \\ 1 & \text { otherwise }\end{cases}
$$

Recall that D (resp. C, O, E) denotes the collection of SNREs which belong to the dominated type (resp. cooperating, oscillating and equal growth types). For general $d, k \in$ $\mathbb{N}$, example study yields that there are far from only 4 types of SNREs. Similar to the case $d=k=2$, we say an SNRE is of the

(1) dominated type if there exists $a \in \mathcal{A}$ such that $a_{n} \geq b_{n}$ for all $b \in \mathcal{A}$;

(2) cooperating type if $\alpha_{n}=\alpha_{n-1}^{d}+g_{n-1}$ with $g_{n} \leq \alpha_{n}^{d}$, where $\alpha_{n}=\sum_{a \in \mathcal{A}} a_{n}$;

(3) oscillating type if for $a, b \in \mathcal{A}$ there exist subsequences $\left\{n_{i}\right\}_{i \geq 1},\left\{m_{i}\right\}_{i \geq 1}$ such that $a_{n_{i}} \geq b_{n_{i}}$ but $a_{m_{i}}<b_{m_{i}}$ for $i \in \mathbb{N}$;

(4) equal growth type if $a_{n}=b_{n}$ for all $a, b \in \mathcal{A}$.

The following theorem indicates that the SNREs corresponding to golden mean shift and $k$-colored chessboard are of the above types.

Theorem 4.1. Let $d, k \in \mathbb{N}$.

(1) If $X_{A}$ is a golden mean shift, then $X_{A}$ is of the type $\mathbf{C} \cap \mathbf{D}$. 
(2) If $X_{A}$ is a $k$-colored chessboard, then $X_{A}$ is of the type $\mathbf{E}$.

Proof. Suppose $X_{A}$ is a golden mean shift. For clarity, we only prove the case where $k=2$, and the other cases can be treated similarly. Let $\mathcal{A}=\{1,2\}$. Since $A$ is defined in 4.1, we have $\mathcal{B}^{(1)}=\left\{\left(1 ; i_{1}, i_{2}, \ldots, i_{d}\right): i_{j}=1\right.$ or 2 for $\left.1 \leq j \leq d\right\}$ and $\mathcal{B}^{(2)}=\{(2 ; 1,1, \ldots, 1)\}$. Thus, the corresponding SNRE with respect to $\mathcal{B}=\mathcal{B}^{(1)} \cup \mathcal{B}^{(2)}$ is of the following form

$$
\begin{gathered}
a_{n}=a_{n-1}^{d}+C_{1}^{d} a_{n-1}^{d-1} b_{n-1}+C_{2}^{d} a_{n-1}^{d-2} b_{n-1}^{2}+\cdots+b_{n-1}^{d}, \\
b_{n}=a_{n-1}^{d}, \quad a_{1}=2^{d} \quad \text { and } \quad b_{1}=1 .
\end{gathered}
$$

It can be easily checked that $F=\left\{F^{(a)}, F^{(b)}\right\}$ is of the type $\mathbf{C} \cap \mathbf{D}$ under the same discussion in Propositions 3.6 and 3.8. Suppose $X_{A}$ is a $k$-colored chessboard. We have $\mathcal{B}^{(i)}=\left\{\left(i ; i_{1}, i_{2}, \ldots, i_{d}\right): i_{j} \neq i\right.$ for $\left.1 \leq j \leq d\right\}$. The corresponding SNRE is of the following form $a_{n}^{(i)}=\left(\sum_{1 \leq j \leq k, j \neq i} a_{n-1}^{(j)}\right)^{d}$. Thus, $F$ is of the type $\mathbf{E}$. The proof is complete.

Corollary 4.2. Let $2 \leq d \in \mathbb{N}$. Then

(1) The entropy of an $S_{d^{-}} G M S$ for $k=2$ is $h\left(X_{A}\right)=\frac{(d-1) A_{\infty}}{d^{2}}$, where

$$
A_{\infty}=\lim _{n \rightarrow \infty} A_{n}=d \ln 2+\lim _{n \rightarrow \infty} \sum_{j=1}^{n-1} d^{-j} \ln r_{j}^{(a)}
$$

$$
\text { and } r_{j}^{(a)}=1+C_{1}^{d} \frac{b_{j}}{a_{j}}+C_{2}^{d}\left(\frac{b_{j}}{a_{j}}\right)^{2}+\cdots+C_{d-1}^{d}\left(\frac{b_{j}}{a_{j}}\right)^{d-1}+\left(\frac{b_{j}}{a_{j}}\right)^{d} .
$$

(2) The entropy of a $k$-colored chessboard is $\ln (k-1)$.

Proof. It follows from Theorem 4.1 that $X_{A}$ is of the type $\mathbf{D}$ and $a$ is the dominating element. Under the identical argument as the proof of Proposition 3.6, we have $h\left(X_{A}\right)=$ $\frac{(d-1) A_{\infty}}{d^{2}}$, where $A_{\infty}=\lim _{n \rightarrow \infty} A_{n}=d \ln 2+\lim _{n \rightarrow \infty} \sum_{j=1}^{n-1} d^{-j} \ln r_{j}^{(a)}$. If $X_{A}$ is a $k$-colored chessboard, its SNRE is of type $\mathbf{E}$. It follows from Proposition 3.3 that $h\left(X_{A}\right)=\ln (k-1)$. This completes the proof.

The method developed in $S_{2}$-GMS can also be applied to the traditional one-dimensional GMS.

Example 4.3. Let $X_{A}$ be the golden mean shift of $(d, k)=(1,2)$. That is, $X_{A}=\{x \in$ $\{1,2\}^{\mathbb{N} \cup\{0\}}: A\left(x_{i}, x_{i+1}\right)=1$ for $\left.i \geq 1\right\}$, where $A=\left(\begin{array}{ll}1 & 1 \\ 1 & 0\end{array}\right)$. The SNRE 4.2$)$ gives

$$
a_{n}=a_{n-1}+b_{n-1}, \quad b_{n}=a_{n-1}, \quad a_{1}=2 \quad \text { and } \quad b_{1}=1 .
$$

Note that 4.3 is an linear recursive equation, it follows from (3.4) we have

$$
\ln a_{n}=A_{n}=\ln 2+\sum_{j=1}^{n-1} \ln r_{j}^{(a)}=\ln 2+\ln \prod_{j=1}^{n-1} r_{j}^{(a)}
$$




$$
=\ln \prod_{j=1}^{n-1} 2\left(1+\frac{b_{j}}{a_{j}}\right)=\ln 2\left(1+\frac{1}{2}\right)\left(1+\frac{2}{3}\right) \cdots\left(1+\frac{b_{n-1}}{a_{n-1}}\right) .
$$

In this case $\left|E_{n}\right|=n$, thus

$$
\begin{aligned}
h\left(X_{A}\right) & =\lim _{n \rightarrow \infty} \frac{A_{n}}{n}=\lim _{n \rightarrow \infty} \frac{\ln a_{n}}{n}=\lim _{n \rightarrow \infty} \frac{1}{n} \ln 2\left(1+\frac{1}{2}\right)\left(1+\frac{2}{3}\right) \cdots\left(1+\frac{b_{n-1}}{a_{n-1}}\right) \\
& =\lim _{n \rightarrow \infty} \frac{1}{n} \ln \left(a_{n-1}+b_{n-1}\right)=\lim _{n \rightarrow \infty} \frac{1}{n} \ln a_{n}=\ln g,
\end{aligned}
$$

where $a_{n}$ is the $n$-th Fibonacci number and $g=\frac{1+\sqrt{5}}{2}$.

\subsection{Golden mean shifts on free groups}

In this section we restrict our discussion to the entropy formula of $F_{d}$-GMSs. The previous results on $S_{d}$-SFTs can be applied to solve the entropy formula of the $F_{d}$-GMS. Let $2 \leq d$, $k=2$, and $q=2 d-1$.

Theorem 4.4. Let $X$ be the $F_{d^{-}} G M S$, then we have $h(X)=\frac{(q-1) A_{\infty}}{q^{2}}$, where

$$
A_{\infty}=q \ln 2+\lim _{n \rightarrow \infty} \sum_{j=1}^{n-1} q^{-j} \ln r_{j}^{(a)},
$$

and $r_{j}^{(a)}=1+C_{1}^{d} \frac{b_{j}}{a_{j}}+C_{2}^{d}\left(\frac{b_{j}}{a_{j}}\right)^{2}+\cdots+C_{d-1}^{d}\left(\frac{b_{j}}{a_{j}}\right)^{d-1}+\left(\frac{b_{j}}{a_{j}}\right)^{d}$.

Proof. Suppose $\mathcal{A}=\{a, b\}$ and let $\widehat{a}_{n}$ (resp. $\widehat{b}_{n}$ ) be the number of configurations of $X$ on $G_{n}=\left\{g \in F_{d}:|g| \leq n\right\}$ with the root $\epsilon$ being colored by 1 (resp. 2). We also denote by $a_{n}$ (resp. $b_{n}$ ) the number of configurations of $X$ on $E_{n}=\left\{g \in S_{q}:|g| \leq n\right\}$ with the root being colored by 1 (resp. 2). Since $q=2 d-1$, we have the following formulae

$$
\widehat{a}_{n}=a_{n-1}^{2 d}+C_{1}^{2 d} a_{n-1}^{2 d-1} b_{n-1}+\cdots+C_{1}^{2 d} a_{n-1} b_{n-1}^{2 d-1}+b_{n-1}^{2 d}, \quad \widehat{b}_{n}=a_{n-1}^{2 d},
$$

and

$$
a_{n}=a_{n-1}^{q}+C_{1}^{q} a_{n-1}^{q-1} b_{n-1}+\cdots+C_{1}^{q} a_{n-1} b_{n-1}^{q-1}+b_{n-1}^{q}, \quad b_{n}=a_{n-1}^{q} .
$$

Let $F=\left\{F^{(a)}, F^{(b)}\right\}$ be the SNRE (4.5), we know that $F$ is of the type $\mathbf{D}$ (Theorem 4.1 . Then we have $\left|E_{n}\right|=\sum_{i=0}^{n} q^{i}=\frac{q^{n+1}-1}{q-1}$ and $\left|G_{n}\right|=1+2 d\left|E_{n-1}\right|$. Since $a$ is a dominating element, it follows from Lemma 3.5 and (4.4) that

$$
\begin{aligned}
h(X) & =\lim _{n \rightarrow \infty} \frac{\ln \left(\widehat{a}_{n}+\widehat{b}_{n}\right)}{\left|G_{n}\right|}=\lim _{n \rightarrow \infty} \frac{\ln \widehat{a}_{n}}{\left|G_{n}\right|}=\lim _{n \rightarrow \infty} \frac{\ln \left(a_{n-1}^{2 d}+\cdots+b_{n-1}^{2 d}\right)}{1+2 d\left|E_{n-1}\right|} \\
& =\lim _{n \rightarrow \infty} \frac{2 d \ln a_{n-1}}{\left|E_{n-1}\right|}\left(\frac{\left|E_{n-1}\right|}{1+2 d\left|E_{n-1}\right|}\right)=\lim _{n \rightarrow \infty} \frac{\ln a_{n-1}}{\left|E_{n-1}\right|} .
\end{aligned}
$$


Proposition 3.6 is applied to show that $\lim _{n \rightarrow \infty} \frac{\ln a_{n-1}}{\left|E_{n-1}\right|}=\frac{(q-1) A_{\infty}}{q^{2}}$, where

$$
A_{\infty}=\lim _{n \rightarrow \infty} A_{n}=q \ln k+\lim _{n \rightarrow \infty} \sum_{j=1}^{n-1} q^{-j} \ln r_{j}^{(a)} .
$$

Thus $h(X)=\frac{(q-1) A_{\infty}}{q^{2}}$. This completes the proof.

\section{Conclusion and open problems}

List results of this paper as follows.

(1) Four types, namely $\mathbf{E}, \mathbf{D}, \mathbf{C}, \mathbf{O}$ types, are introduced. Their entropy formulae are presented in Propositions 3.3, 3.6, 3.8, and 3.10 respectively. Furthermore, the set of all SNREs with $(d, k)=(2,2)$ is equal to $\mathbf{E} \cup \mathbf{D} \cup \mathbf{C} \cup \mathbf{O}$ (Section 3.5). This gives a complete characterization for the entropies of $S_{2}$-SFTs with two symbols.

(2) Two types of hom-shifts on $S_{d}$, the $S_{d}$-GMS and $k$-colored chessboard on $S_{d}$, are introduced. The entropy formulae of these two types are presented (Corollary 4.2). The entropy formula of the $F_{d}$-GMS is also developed by using the method of entropy theory on $S_{d}$-SFTs (Theorem 4.4).

The formulas addressed are presented as geometric series, which provide another evidence of the existence of the topological entropy of $S_{d}$-shift spaces, despite the shifts we consider is only a small class. On the other hand, these geometric series are useful for estimating the topological entropy of shift spaces which are approximated by the shifts considered in this elucidation. Although we give the characterization for $(d, k)=(2,2)$, the general entropy formula for arbitrary $(d, k) \in \mathbb{N} \times \mathbb{N}$ is far from being solved. We list some possible problems in the future study.

Problem 5.1 (Priori criterion for an SNRE). In Section 3, we introduce four types of SNRE on which we can compute their entropies explicitly. But the following criteria seem more important for $|\mathcal{A}| \geq 3$.

(1) How to check if $F$ admits a dominating element?

(2) How to check if $F$ belongs to the oscillating type?

(3) Can we characterize all SNREs completely for arbitrary $|\mathcal{A}|$ ?

Problem 5.2 (Zero order estimate). Let $X^{\mathcal{B}}$ be an $F_{d}$-SFT or $S_{d^{-}}$SFT. Recall that the value $\kappa$ (resp. $\lambda_{2}$ and $\lambda_{1}$ ) is the $2^{n d}$-order (resp. $1^{\text {st }}$-order and zero-order) speed of $B_{n}\left(X^{\mathcal{B}}\right)$ (see $(1.2)$ ). We have shown so far that there exists an algorithm to compute $\kappa\left[3\right.$ and $\lambda_{2}$. 
However, the number $\lambda_{1}$ seems crucial for the accuracy of the number $\left|B_{n}\left(X^{\mathcal{B}}\right)\right|$. In $[16]$, the author shows that the value $\lambda_{1}$ may not exist in the case of $F_{d}$-GMS. We may ask: under which conditions $\lambda_{1}$ exists, and how to compute it? Another question is whether there exists an intermediate order between $\lambda_{1}$ and $\lambda_{2}$.

Problem 5.3 (Entropy of SFTs on free groups). In Section 4, we study the entropy of $F_{d}$-GMS. However, the method can not apply to general $F_{d}$-SFTs. One of the reasons is that the SNRE (4.4) and (4.5) depend on the length of $E_{n}$ of the groups $S_{q}$ (but the case of $F_{d^{-}}$GMS will not). So, what is the entropy formula for an $F_{d}$-SFT? Furthermore, if $G$ is a group which is not free, how to compute the entropy of the $G$-SFT?

\section{Acknowledgments}

We want to express our gratitude to the anonymous referees. Their valuable comments and suggestions have significantly improved the readability and quality of this paper. Also, some inspired further study is under preparation.

\section{References}

[1] N. Aubrun and M.-P. Béal, Tree-shifts of finite type, Theoret. Comput. Sci. 459 (2012), 16-25.

[2] J.-C. Ban and C.-H. Chang, Tree-shifts: Irreducibility, mixing, and the chaos of treeshifts, Trans. Amer. Math. Soc. 369 (2017), no. 12, 8389-8407.

[3] _ Tree-shifts: The entropy of tree-shifts of finite type, Nonlinearity 30 (2017), no. $7,2785-2804$.

[4] _ Characterization for entropy of shifts of finite type on cayley trees, J. Stat. Mech. Theory Exp. 2020 (2020), no. 7, 073412-73431.

[5] R. Bowen, Equilibrium States and the Ergodic Theory of Anosov Diffeomorphisms, Lecture Notes in Mathematics 470, Springer-Verlag, Berlin, 1975.

[6] N. Chandgotia and B. Marcus, Mixing properties for hom-shifts and the distance between walks on associated graphs, Pacific J. Math. 294 (2018), no. 1, 41-69.

[7] S. Friedland, Multi-dimensional capacity, pressure and Hausdorff dimension, in: Mathematical Systems Theory in Biology, Communications, Computation, and Finance (Notre Dame, IN, 2002), 183-222, IMA Vol. Math. Appl. 134, Springer, New York, 2003. 
[8] G. A. Hedlund, Endomorphisms and automorphisms of the shift dynamical system, Math. Systems Theory 3 (1969), 320-375.

[9] M. Hochman and T. Meyerovitch, A characterization of the entropies of multidimensional shifts of finite type, Ann. of Math. (2) 171 (2010), no. 3, 2011-2038.

[10] S. A. B. Lemp, Shift Spaces on Groups: Computability and ynamics, Ph.D. thesis, Université de Lyon, 2017.

[11] D. A. Lind, The entropies of topological Markov shifts and a related class of algebraic integers, Ergodic Theory Dynam. Systems 4 (1984), no. 2, 283-300.

[12] D. Lind and B. Marcus, An Introduction to Symbolic Dynamics and Coding, Cambridge University Press, Cambridge, 1995.

[13] B. Marcus and R. Pavlov, Approximating entropy for a class of $\mathbb{Z}^{2}$ Markov random fields and pressure for a class of functions on $\mathbb{Z}^{2}$ shifts of finite type, Ergodic Theory Dynam. Systems 33 (2013), no. 1, 186-220.

[14] _ Computing bounds for entropy of stationary $\mathbb{Z}^{d}$ Markov random fields, SIAM J. Discrete Math. 27 (2013), no. 3, 1544-1558.

[15] K. Petersen and I. Salama, Tree shift topological entropy, Theoret. Comput. Sci. 743 (2018), 64-71.

[16] S. T. Piantadosi, Symbolic dynamics on free groups, Discrete Contin. Dyn. Syst. 20 (2008), no. 3, 725-738.

[17] D. Ruelle, Thermodynamic Formalism: The mathematical structures of classical equilibrium statistical mechanics, Addison-Wesley, Reading, Mass., 1978.

Jung-Chao Ban

Department of Mathematical Sciences, National Chengchi University, Taipei 11605,

Taiwan

E-mail address: jcban@nccu.edu.tw

Chih-Hung Chang

Department of Applied Mathematics, National University of Kaohsiung, Kaohsiung 81148, Taiwan

E-mail address: chchang@nuk.edu.tw 